MEI, II, Vol. 2, nº 3, pág. 99

\title{
Babel de papel: las bibliotecas del Instituto Cervantes como puntos de encuentro multiculturales \\ MEI
}

II, vol. 2

$\mathrm{n}^{0} 3$

Recibido el 23-11-2011

Aceptado en $31-12-2011$

\author{
Begoña Colmenero Niño \\ Jefa de Biblioteca del Instituto Cervantes de Brasilia
}

\section{Resumen}

Las bibliotecas del Instituto Cervantes tienen como objetivo romper estereotipos y acabar con los prejuicios culturales. Pretenden ser lugares donde se muestre el lado menos conocido de nuestra cultura y nuestra civilización; pero también donde se busquen puntos comunes y se establezcan contactos personales que ayuden al acercamiento mutuo a través de iniciativas que abarquen aspectos tan diversos como la música, el cine, la gastronomía, la literatura, las tradiciones, el arte, etc.

Se explican algunas de las experiencias más importantes llevadas a cabo en ese sentido en los centros situados en los países del Mediterráneo en las que se reflejan cómo las bibliotecas del Instituto Cervantes se convierten día a día en puentes culturales por el que sus usuarios transitan en un viaje continuo de ida y vuelta.

\section{Palabras clave}

Instituto Cervantes; Multiculturalismo; Cultura hispánica, Divulgación.

Babel paper : Instituto Cervantes libraries as a multicultural meeting point

Abstract

The libraries of Instituto Cervantes aims to eliminate stereotypes and cultural prejudices. Intended to be places that show the lesser known side of our culture and civilization; but also where to seek common ground and establish personal contacts to help the rapprochement through initiatives covering areas as diverse as music, cinema, gastronomy, literature, traditions, art, etc.

Some of the most important experiences carried out in this sense are explained in the Centers of the Mediterranean countries in which reflects how the libraries of the Instituto Cervantes become a daily cultural bridges where users transit in an ongoing journey back and forth.

\section{Keywords}

Cervantes Institute; Multiculturalism; Hispanic culture, Divulgation.

\section{Introducción}

El concepto de biblioteca ha variado enormemente en los últimos años. Hasta hace poco eran concebidas fundamentalmente como depósitos de libros, salas de estudio o como mucho, en el caso de las bibliotecas ligadas a instituciones de enseñanza, como centros de apoyo a la enseñanza formal.

Aunque las bibliotecas han jugado un siempre un papel social básico, puesto que al garantizar el acceso gratuito e igualitario a la información se convierten en un espacio democrático, libre y abierto, ha sido especialmente en los últimos años, en la medida en la que la sociedad ha evolucionado hacia la llamada sociedad de la información y las demandas 
de los usuarios han cambiado cuando las bibliotecas han diversificado sus funciones, su estructura y sus servicios y se han visto obligadas a hacerlo al mismo ritmo vertiginoso al que lo hace la sociedad.

Hoy en día la información se ha convertido es uno de los bienes más valiosos que posee el hombre y las bibliotecas son los centros que la albergan, la procesan, la gestionan y la difunden. Por eso las bibliotecas juegan un papel social cada vez más importante.

Las bibliotecas son actualmente instituciones públicas de enseñanza permanente, en el que se produce un intercambio continuo de información, son centros sociales en los que los usuarios buscan mucho más que satisfacer una demanda de información, sino un espacio de participación ciudadana, en el que entrar en contacto con otras culturas, divertirse, en el que crecer como personas.

Vivimos en la era de las comunicaciones. Debido al increíble desarrollo tecnológico de los últimos años se ha producido un intercambio de información, bienes y personas que han dado lugar a otro de los grandes fenómenos del siglo $\mathrm{XX}$, el de la globalización.

Cada vez es mayor el interés por conocer al otro, por ampliar horizontes, aprender otros idioma, descubrir culturas distintas a la nuestra. En ese sentido las bibliotecas se ven obligadas una vez más a adaptarse a la demanda de un público que en muchas ocasiones ya no se conforma con tener acceso a la cultura local, con fondos en su propio idioma, sino que la biblioteca se convierte en el punto de acceso, a través de la literatura, la música, el cine, el arte, etc., a otros idiomas, a otras culturas exóticas y lejanas a la nuestra.

Por otro lado nuestras ciudades y nuestros países son cada vez menos homogéneos. El fenómeno de la inmigración afecta en mayor o menor medida y por motivos diferentes a cada vez más países y la población es cada vez más variada y multiétnica.

\section{La RBIC: Red de bibliotecas del Instituto Cervantes}

En medio de ese panorama de cambios, revolución tecnológica y globalización, aparece, hace ahora 20 años la red de bibliotecas del Instituto Cervantes. Esta institución surgió en 1991 con la intención de promover la enseñanza, el estudio y el uso del español y difundir la cultura de los países y pueblos de la comunidad hispanohablante.

Comenzó tímidamente pero en su corto periodo de vida se ha extendido a un ritmo tal que en la actualidad cuenta ya con 77 centros distribuidos en 34 países y 5 continentes. Cuenta con una red de 62 bibliotecas, que al igual que la propia institución está en continuo crecimiento desde su creación. Se trata, pues, de la mayor red de bibliotecas españolas en el mundo. 
Si observamos la distribución geográfica de estos centros vemos que, aunque en la actualidad se está expandiendo fundamentalmente por América y Asia, por razones históricas la mayor concentración de centros se encuentra en el área mediterránea, donde se cuenta con 21 bibliotecas situadas en Ammán (Jordania), Argel y Orán (Argelia), Atenas (Grecia), Beirut (Líbano), Casablanca, Fez, Tetuán, Rabat, Marrakech, Tánger - una de las bibliotecas más antiguas de la red (Marruecos), Damasco (Siria), El Cairo (Egipto), Estambul (Turquía), Madrid (España), Milán, Roma, Nápoles, Palermo (Italia), Tel-Aviv (Israel) y Túnez (Túnez).

El origen de estas bibliotecas es tan diverso como el de los países en los que se encuentran. En aquellos que históricamente han tenido alguna relación con España, Norte de África, mundo árabe y Filipinas especialmente, se absorbieron las bibliotecas de los centros culturales y educativos que ya existían en el exterior, trayendo consigo sus colecciones, instalaciones, servicios y personal. En otros casos son totalmente de nueva creación y su apertura supone en muchos casos el primer desembarco de una institución cultural española en el país.

Por lo tanto, nos encontramos con bibliotecas que, aunque persiguen un objetivo común, responden a perfiles muy heterogéneos, debido tanto a sus orígenes diversos como a las diferencias de sus países anfitriones en aspectos tan diversos e importantes como el nivel de desarrollo, su tradición bibliotecaria, el número de lenguas habladas, su tradición colonial o relación histórica con España, etc.

Así pues, algo que caracteriza a la red de bibliotecas del IC es que ya, a diferencia de otras bibliotecas que han tenido que adaptarse y reciclarse ante el fenómeno de la diversidad cultural, esta red ya nace con un espíritu multicultural.

\section{El carácter multicultural de la RBIC.}

\section{Fondo bibliográfico: un viaje por múltiples leguas y culturas sin moverse de la biblioteca.}

El fondo bibliográfico de la RBIC es un claro exponente del carácter multicultural del que estamos hablando. Está formado por más de un millón de volúmenes, abarcando desde la cultura actual y clásica hasta materias tan variadas como la música, el arte, la historia, la literatura, la arquitectura, etc. Con ella se pretende ofrecer una visión amplia, actual, objetiva y variada de la cultura española así como servir a las necesidades de información de un público variado que cambia y se renueva continuamente.

La lengua predominante es el español pero existen también numerosas obras en el resto de lenguas oficiales del estado español (gallego, vasco y catalán). Debido a su dimensión internacional y a su condición de lugar de encuentro multicultural, los fondos combinan obras publicadas dentro y fuera de nuestras fronteras.

Una de sus secciones más importantes, tanto por el número de volúmenes como por el de préstamos, son las de lengua y literatura, el material de enseñanza de español. Estas secciones cumplen con el objetivo de servir de apoyo a la actividad docente del 
centro, pero también tienen un gran valor para la formación autodidacta del español entre personas de bajos recursos económicos y mantienen vivo el conocimiento de la lengua de aquellos que ya lo han aprendido.

La sección local es otra sección clave en cualquier biblioteca cervantina. Constituye el verdadero enlace entre España y el país en el que está enclavada la biblioteca puesto que en esta sección se recogen:

- obras sobre España y traducciones de literatura española e Hispanoamericana al idioma local; aquí se recogen obras que difícilmente se pueden conseguir en España y gracias a las cuales llegamos a un público que está interesado en conocer nuestra cultura y que pueda acceder a ella en su propio idioma.

- obras publicadas en cualquier lengua del estado español sobre el país donde la biblioteca está ubicada y traducciones de su literatura. Este fondo es muy consultado por la comunidad hispanohablante del país que en ocasiones no conoce el idioma local; también constituye un fondo de referencia para otras bibliotecas e instituciones españolas que recurren a él habitualmente cuando organizan determinadas actividades sobre el país anfitrión.

- obras sobre las relaciones políticas, históricas, económicas... entre los dos países en cualquiera de sus idioma. Es aquí donde están encuadradas algunas de las colecciones especiales que se han ido desarrollando en las distintas bibliotecas y que no podemos dejar pasar por alto. Una de las más destacadas es la dedicada a África y mundo árabe de la biblioteca de Tánger, una de las mejores colecciones sobre la historia de España en el norte de África durante los últimos siglos, formada por unos 9000 documentos donde se recoge literatura colonial producida en Marruecos en la zona del Protectorado Español, Protectorado Francés y en la zona internacional de Tánger, una sección de hispano-arabismo con obras sobre Al Andalus, literatura arábigo-andaluza, en español y en árabe, obras sobre Marruecos, Ceuta, Melilla, Gibraltar, Zona del Estrecho, Canarias, ex colonias de Ifni, Sáhara y Guinea Ecuatorial y obras de tema tangerino de gran valor ya que muchas de ellas son ejemplares únicos. Esta colección se completa con algunos fondos existentes en la biblioteca de Rabat y en la de Tetuán.

También es destacable la colección Sefarad, donde se reúnen aquellos documentos que tienen relación con el judaísmo y España o con los judíos que tuvieron que abandonar Sefarad tras el decreto de expulsión en 1492 (por lo tanto con el ladino en todas sus manifestaciones y en cualquier parte del mundo). Actualmente forman esta colección unos ejemplares pertenecientes a las bibliotecas del Cervantes de Tel Aviv y de Estambul. En este último centro lo que ha tenido más peso a la hora de seleccionar las obras ha sido el sefardita o ladino, lengua muy activa en la ciudad de Estambul y que se conserva como un tesoro en las familias que aún la hablan.

No podía faltar una colección dedicada la obra más universal y más traducida de la literatura española. Traducciones del Quijote es una colección que se creó con motivo de del cuarto centenario de la primera edición del Quijote y en la actualidad cuenta con cerca de 200 obras en las siguientes lenguas: albanés, alemán, árabe, búlgaro, catalán, 
checo, chino, coreano, croata, danés, esloveno, esperanto, estonio, euskera, finés, francés, gallego, georgiano, griego, hebreo, hindi, holandés, húngaro, inglés, irlandés, islandés, italiano, japonés, latín, lituano, maltés, noruego, persa, polaco, portugués, rumano, ruso, serbio, sueco, tagalo, tailandés, turco, vietnamita y yiddish. Esta exposición en la actualidad se encuentra disponible en la biblioteca del Instituto Cervantes de Madrid, pero existe también un catálogo virtual de su fondo.

Por último citar colecciones como Fortificaciones españolas en Orán , ubicada en la biblioteca de esa misma ciudad, el Fondo AISPI, donde se recogen todas las publicaciones de la Asociación de Hispanistas Italianos que fueron donadas a la biblioteca de Roma o Imagen de España, en la que se pretende analizar y mostrar cómo nos han visto y nos ven en el extranjero. Esta colección, de la biblioteca de la sede en Madrid, incluye las crónicas de viajeros por España de todos los tiempos, estudios sobre nuestra imagen de prestigiosos hispanistas como Kamen, Thomas, Inman Fox y estudios de nuestras relaciones internacionales por países. Autores como Orwell, Andersen, Washington Irving, Dumas están también representados a partir de obras donde narran su paso por nuestro país.

También de ese intento de buscar puntos comunes entre ambos países y de singularizar cada una de las bibliotecas de la red surge la idea de bautizar a cada una de ellas con el nombre de un autor que generalmente ha tenido una relación especial con la ciudad o el país. Así nos encontramos con casos como la biblioteca Max Aub de Argel, Vicente Aleixandre de Tetuán o María Zambrano en Roma. Las bibliotecas bautizadas se encargan dar conocer su vida y su obra, de adquirir un fondo especial dedicado a ellos tanto en español como en el idioma local y de hacer actividades para dar a conocer a estas personas que son claros exponentes del mestizaje de culturas al que estamos expuestos hoy en día y que intentan reflejar nuestras bibliotecas en estas secciones.

En los últimos años han aumentado considerablemente el número de recursos de información electrónica disponibles a través de nuestras páginas web. Se trata de un servicio especializado cada vez más solicitado porque proporciona datos y contenidos rigurosos y de calidad, tanto de carácter académico como científico a usuarios potenciales que de otro modo no podrían conseguir, por vivir en un entorno local que en ocasiones es precario tanto tecnológica como académicamente.

Los recursos de información en línea no sólo convierten a las bibliotecas del Instituto Cervantes en proveedoras de información digital en español y sobre el español, sino que también acercan a los usuarios las tecnologías digitales vigentes. A través de ellos se garantiza la libertad de acceso a la información para todos sus usuarios.

Por todo ello este servicio es de gran utilidad pero está especialmente valorado en países con un desarrollo bajo o inexistente de fondos, instalaciones y servicios bibliotecarios, ya que sirve para paliar alguna de esas carencias. 


\section{Actividades: participación, diversión y enriquecimiento para todos}

Todos tenemos estereotipos y prejuicios generalmente debidos al desconocimiento. Aunque poco a poco la imagen de España va cambiando en el mundo todavía, muy a nuestro pesar, sigue habiendo mucha gente que la asocia al flamenco, los toros, al sol y playas. Cuando preguntamos fuera de nuestras fronteras en muchas ocasiones nos damos cuenta de lo limitados que son los referentes sobre nuestro país en el extranjero.

En cuestión de literatura los nombres más conocidos son Lorca o Cervantes y si hablamos de arte, cine o música nos encontraremos con Gaudí, Picasso, Almodóvar o Julio Iglesias, pero hay demasiadas personas que no conocen mucho más sobre nosotros. Nuestra misión consiste en ampliar esa imagen, dar a conocer nuevos nombres que a pesar de no ser universales tienen qué carecer de interés.

No podemos olvidar que en muchos países nuestros centros son el único vínculo con la cultura española, tanto para los locales interesados en ella como para los expatriados españoles y la principal fuente de información nuestro país en el extranjero.

Para poder acabar con los prejuicios culturales, con los estereotipos y el desconocimiento necesitamos, no sólo apoyar a la actividad cultural del centro, sino también desarrollar un programa de actividades propias (visitas guiadas, tertulias, exposiciones, presentaciones de libros, talleres, concursos, conciertos, debates, proyecciones de películas) en las que nuestros usuarios se sientan involucrados.

Es en estas actividades donde el factor humano entra en juego y donde nuestras bibliotecas alcanzan un valor añadido que sólo pueden aportar la interacción, el intercambio y la comunicación de conocimientos y experiencias.

Por eso además de tener un fondo variado y amplio, como ya indicamos anteriormente, necesitamos saber mostrarlo. No debemos esperar a que sea el usuario el que lo descubra, porque si actuamos así corremos el riesgo de que se limiten a aquello que ya es familiar para ellos. Existen muchas formas de conseguirlo y como demostración relataré algunas de las más interesantes llevadas a cabo en las bibliotecas de la RBIC del área mediterránea.

Una de las actividades que mejor funciona son las exposiciones bibliográficas de una parte de nuestra colección que por alguna razón se considera importante mostrar, como por ejemplo se hizo con la exposición de las Traducciones del Quijote”, o de muestras de una serie de obras, generalmente literarias, en español y el idioma local, como por ejemplo se hizo con la exposición "Del español al turco y del turco al español" organizada en 2003 por la biblioteca del IC de Estambul, que se expuso en Estambul, Ankara y Bursa en colaboración con distintas bibliotecas locales.

Otra buena experiencia fue la exposición "Ex libris: Red de Bibliotecas del Instituto Cervantes", donde se mostraban que se clausuró con la I Jornada Profesional de la 
RBIC en la que se reunió a expertos internacionales en colecciones españolas, con el propósito de iniciar una línea de trabajo que se promueva el intercambio entre profesionales españoles y extranjeros.

Las tertulias literarias son una práctica generalizada en muchas bibliotecas cervantinas. Por un lado a las personas que están aprendiendo nuestro idioma se les ofrece un espacio donde practicarlo y estar en contacto con los nativos que acuden a estas actividades.

Por otro, son el medio de descubrir autores o aspectos de la literatura menos conocidos y por último son un foro de discusión e intercambio de ideas, donde todo el mundo se enriquece. En muchas ocasiones sirven para hacer estudios comparativos de distintos aspectos de la tradición literaria del país anfitrión con el nuestro u otros países, en otros sirven para comparar ideas sobre temas que interesan a todos los interlocutores. Por ejemplo, buscar personajes comunes en la literatura tradicional árabe y la española o encontrar las geografías imaginarias en la literatura mediterránea.

La tradición oral es otra de las formas de implicar a los usuarios en el mundo de la biblioteca en general y de los libros en concreto. Actividades tan básicas como reunir a nuestros usuarios y dejar que cada uno cuente una anécdota, un cuento, una adivinanza o un trabalenguas de su propia tradición son tan enriquecedoras y sencillas que tienen muchísima aceptación.

Un recurso muy utilizado son los concursos literarios, donde se suelen buscar temas que sean el símbolo del mestizaje que se busca con estas actividades. Por un lado los usuarios tienen la oportunidad de mostrar su creatividad y capacidad literaria, en otros también sus conocimientos lingüísticos, ya que muchas veces se requieren que se expresen en español.

Un ejemplo de esa experiencia es el "Concurso literario de cuentos tunecinos de tradición oral" que convocó en el Instituto Cervantes de Túnez en abril de 2007 con motivo de la celebración del Día Internacional del Libro, donde los participantes presentaron un cuento inédito de la tradición oral tunecina, en árabe dialectal tunecino y español que leyeron posteriormente en un acto público.

El cine es otro campo que suele despertar mucho interés. Además de los clásicos proyecciones de películas en la lengua del país o de los múltiples ciclos de temática de actualidad, se han desarrollado iniciativas tan curiosas como animar a los alumnos a que seleccionen un fragmento de una película, lo traduzcan al español y lo doblen. Si después se culmina con la proyección del experimento esta actividad tiene el éxito asegurado.

¿Y por qué no organizar un concurso de cocina en el que los concursantes tienen que redactar la receta y con todas ellas publicar un libro en español que ayude a nuestros estudiantes a mostrar los conocimientos de nuestro idioma y a muchas personas a descubrir las delicias culinarias de los países mediterráneos? 
En muchas ocasiones nuestros usuarios no están muy acostumbrados a utilizar nuestro catálogo o desconocen el funcionamiento de nuestra biblioteca y los servicios que se le ofrecen.

Para acercarla a ella se organizan visitas guiadas en su propio idioma que con casi de obligado cumplimiento con todos los nuevos alumnos que se matriculan en el centro, pero que se ofrecen también a todos los nuevos visitantes. Como parte también de nuestra misión como centro de formación, alfabetización informacional y aprendizaje permanente se organizan sesiones especiales en las que se muestran recursos dedicados a un sector especial de nuestros usuarios, como por ejemplo profesores de español, o que pretenden mejorar determinadas destrezas como, por ejemplo "Cómo iniciar una investigación” o "Utilización de recursos de información electrónica”.

La hora del cuento, talleres de expresión y literatura, de ilustración, charlas, participación en ferias del libro, conferencias, presentaciones de libros... son tantas las formas de conocer al otro, de ampliar horizontes y sorprendernos a nosotros mismos descubriendo todo lo que nos queda por aprender.

\section{La biblioteca a la carta: los productos documentales.}

Los productos documentales complementan tanto a las actividades como a las colecciones. Cada biblioteca cuenta con su propia página web y su guía en papel que tiene que estar como mínimo en el idioma del país y en español.

Quizá el producto documental estrella es nuestro catálogo colectivo en línea desde donde se puede acceder a la información de toda nuestra colección. No podemos olvidar que al tratarse de una red de bibliotecas, independientemente de dónde estén ubicados estos fondos todos forman parte de un mismo catálogo común que, gracias al servicio de préstamo interbibliotecario, los hace accesibles a los usuarios en cualquiera de los países donde tengamos una sede. Muchas veces a los usuarios que no están familiarizados con nuestra producción artística o cultura y les resulta difícil elegir seleccionar el libro o una película.

Para ello son muy útiles guías temáticas como la de "Películas que tratan el tema de la emigración / inmigración desde el punto de vista de España, África y Latinoamérica" o la "Guía de lectura de dariya o árabe dialectal marroquî" elaboradas en la biblioteca de Tetuán.

Estas guías a veces tienen un sentido más práctico y proporcionan datos o consejos sobre temas tan recurrentes como la homologación de títulos universitarios, pasos para acceder a la universidad española o guías de estudios en España.

Una experiencia más reciente son los dossieres temáticos que prepara el Departamento de Documentación con sede en Madrid y que están disponibles en línea. Estos surgen de la necesidad de responder a demandas de información que se repiten en casi todos los países por tratarse de temas que interesan muchísimo a casi todos nuestros usuarios. La mayoría de estos dossieres, como el que informa sobre 
Becas para extranjeros en España, sobre la Situación del español en el mundo o sobre la Imagen de España, por citar algunos, han demostrado tener muchísima utilidad y aceptación, e incluso se han anticipado en algunas ocasiones a las necesidades de nuestros visitantes.

Por último no podemos olvidar las ventajas de la aparición e incorporación de las tecnologías de la información y la comunicación (TIC) que nos ofrecen la posibilidad de crear redes y comunidades más amplias, eliminando las barreras físicas que eran un obstáculo hasta hace poco. Con el uso de herramientas como Facebook, Twitter, blogs, etc. la biblioteca se ha vuelto más participativa y la comunicación e intercambio de información con nuestros usuarios es mucho más fluida y constante, lo que nos ayuda a conocerlos mejor.

La gran ventaja de las redes sociales es que al ser gratuitas, muy flexibles y fáciles de usar, tanto para los usuarios como para los bibliotecarios que las administran, son muy utilizadas, de forma que es un vehículo que aumenta la presencia y la visibilidad de nuestro centro en la web y permite llegar a muchísima gente, incluso a quienes habitualmente no frecuentan una biblioteca. A través de ellas se crean vínculos entre personas que ni siquiera se conocen entre ellas pero que comparten inquietudes comunes. Por ejemplo, el blog de la biblioteca de Londres o de San Paulo puede interesar igualmente a un búlgaro que a un francés o ¿porqué no? a un griego. Los comentarios dejados en Twitter por el bibliotecario de Nápoles pueden resultar muy prácticos a un lector de Sidney o los enlaces recomendados en De.li.ci.us por la biblioteca de Tokio ser de gran valor para una persona que los consulta desde Recife.

\section{El factor humano: la interacción entre bibliotecarios y usuarios}

Otra de las piezas clave en la RBIC es su personal. Los bibliotecarios son junto con los usuarios los que proporcionan a estas instituciones un carácter especial. El factor humano es determinante para conseguir que las bibliotecas sean un lugar abierto, acogedor y agradable al que apetezca volver.

La atención al público es esencial en nuestro trabajo diario, por eso es básico que los responsables de las bibliotecas $\mathrm{y}$, sobre todo, todas las personas que tratan habitualmente con el público, además de tener una buena preparación técnica y profesional, sean personas hospitalarias, comunicativas y tolerantes.

Es muy importante que sean capaces de dirigirse a los usuarios en su propio idioma, tanto para facilitar la comunicación como para romper la timidez o incluso la vergüenza que tienen quienes nos visitan por primera vez. Tienen que saber aceptar y superar las posibles diferencias culturales y adaptar las normas de funcionamiento y el reglamento de la biblioteca a las costumbres del país.

Ellos son los que tienen que aplicar los valores y principios que rigen la actividad y la gestión de la Red de bibliotecas convirtiéndose en agentes de cambio que creen bibliotecas comprometidas con la sociedad, lugares de encuentro donde se propicie el 
diálogo intercultural y un ambiente participativo de todos aquellos que se sienten parte de ella.

Una gran parte de los trabajadores de la RBIC son extranjeros en el país donde trabajan y han experimentado por sí mismos lo que supone adaptarse a una nueva ciudad, aprender otras lenguas, conocer culturas diferentes y valorar todo lo que ello aporta al enriquecimiento personal. Por eso constituyen un elemento muy valioso, porque su experiencia y su bagaje cultural y personal son esenciales a la hora de gestionar una biblioteca de estas características.

Otra de las cualidades del personal de la RBIC es su capacidad de cooperación, ya que trabajan en una red formada por un equipo de profesionales que comparten recursos, experiencias y conocimiento. Además desarrollan un papel básico como enlace entre las instituciones bibliotecarias, educativas y culturales locales y España ya que entre otras tareas promueven y supervisan el intercambio de profesionales de la información entre España y el país o la zona geográfica en la que se encuentran, asesoran en la gestión o creación de redes de bibliotecas en países carentes de infraestructura bibliotecaria, gestionan becas y ayudas para la formación profesional y forman parte de asociaciones internacionales en las que se trabaja por el desarrollo, modernización y mejora de las bibliotecas del país anfitrión. Por citar algunos ejemplos formamos parte del Comité para el Apoyo de bibliotecas de Grecia (CLS), Innovative Library Initiatives Promotion Group (ILIPG) de Estambul o el Egyptian Library Association International Committee (ELIA).

No puedo terminar este artículo sin nombrar a nuestros usuarios, ya que es para ellos para los que trabajamos y sin los que todos nuestros esfuerzos no tendrían sentido. Por la RBIC pasan más de 700.000 visitantes anuales y se atiende a las necesidades de información de más de 56.000 usuarios. Todas estas personas tiene algo en común puesto que todas ellas buscan en nuestras bibliotecas un referente cultural, un lugar donde incrementar su conocimiento, un espacio de convivencia donde ser aceptados independientemente de sus ideas, religión, nacionalidad, idioma o sus ideas.

Muchos de ellos buscan poder practicar el idioma que están aprendiendo, otros encontrar a personas que vienen de los países que les gustaría visitar, del que son originarios o donde tienen un familiar viviendo. Estudiantes, periodistas, arquitectos, diplomáticos, vendedores del Gran Bazar, expatriados, guías turísticos, profesores de español, traductores, filólogos, hispanistas, amantes de flamenco y la salsa, alemanes, brasileños, italianos, tunecinos, japoneses, húngaros, marroquíes, austriacos, españoles, egipcios, israelitas,... todos tienen cabida, todos tienen algo que aportarnos y con todos ellos debemos compartir nuestro idioma nuestra riqueza artística y cultural ya que así conseguiremos crear verdaderos espacios sociales y compartir el sentimiento de pertenecer a una misma comunidad. 
MEI, II, Vol. 2, nº 2, pág. 109

\section{Bibliografía}

ABAD HIRALDO, Ramón. Embajadas Culturales: las Bibliotecas del Instituto Cervantes en su contexto". Métodos de información. No 49, vol. 9, enero-marzo (2002), p. 39-44.

BYRNE, Alex. En: 73th IFLA General Conference and Council. "Libraries for the future: Progress, Development and Partnerships". 19-23 de agosto 2007, Durban, South Africa. La Haya: IFLA, 2007. 7 p.

COLMENERO NIÑO, Begoña. "Türkiye’deki Ispanyol Kültürü: Istanbul Cervantes Enstitüsü Alvaro Mutis Kütüphanesi" = Cultura española en Turquía: La Biblioteca Álvaro Mutis del Instituto Cervantes de Estambul. En: Türk Kütüphaneciligi, 20, 2 (2006), p. 321-328.

COLMENERO NIÑO, Begoña. Biblioteca Álvaro Mutis de Estambul". En: Revista Instituto Cervantes. Año II. No 9, (marzo-abril 2006) p. 44

FORD, Barbara J. "Todos bienvenidos a la biblioteca pública como espacio de integración ciudadana”. En: La Biblioteca Pública, Portal de la Sociedad de la Información. Actas del I Congreso Nacional de Bibliotecas Públicas. Valencia, 29, 30 y 31 de Octubre de 2002. Madrid: MCU, Subdirección General de Coordinación Bibliotecaria, 2002 p. 190-197.

FOURIE, Ina. "Public libraries addressing social inclusion: how we may think...". En: 73th IFLA General Conference and Council. "Libraries for the future: Progress, Development and Partnerships". 19-23 de agosto 2007, Durban, South Africa. La Haya: IFLA, 2007. 9 p.

GARCÍA-ROMERAL PÉREZ, Carlos. "La biblioteca pública y la gestión de la colección: multilingüismo y multiculturalidad". En: IV Jornadas Bibliotecarias de la Comunidad de Madrid. Universidad Carlos III (Madrid), del 22 al 24 de junio de 2004. Madrid: Servicio Regional de Bibliotecas y del Libro, 2004. Pp. 263-273.

GITNER, Fred J. y STUART A. Rosenthal. "Reaching immigrant populations: serving the culturally and linguistically diverse, the Queens Library model”. Bid: textos universitaris de biblioteconomia $i$ documentació, n. 21 , desembre de 2008.

GÓMEZ HERNÁNDEZ, José Antonio. "Biblioteca e integración: de la extensión bibliotecaria a los procesos de inclusión social y digital”. En: De volcanes llena. Biblioteca y compromiso social. P. 343371.

IGLESIA SÁNCHEZ, Yolanda de la, PÉREZ RODRÍGUEZ, Laura. El servicio de acceso a recursos electrónicos en la Red de Bibliotecas del Instituto Cervantes". Bid: textos universitaris de biblioteconomia $i$ documentació, n. 21, desembre de 2008.

KEMPSTER, Grace: "Valuing difference the British Council experience of connecting cultures". En: 69th IFLA Council and General Conference. Berlin, Germany, August 1-9, 2003. La Haya: IFLA, 2003.Manifiesto IFLA por la biblioteca multicultural. La Haya: IFLA, 2008. 4 p.

MARTÍN-MONTALVO, Irene. "La Red de Bibliotecas del Instituto Cervantes: Origen y evolución". Métodos de información. n 49, vol. 9, enero-marzo (2002), p. 32-38.

MARTÍN-MONTALVO, Irene. " Estudio de satisfacción de usuario en la Red de Bibliotecas del Instituto Cervantes". Revista general de información y documentación. 2007, 17, núm. 2, p. 9-30.

PIRSICH, Volker. Nuevas tendencias de los servicios bibliotecarios en comunidades multiculturales en Alemania”. Mesa redonda sobre Bibliotecas Multiculturales organizadas por la Biblioteca Pública Manuel Alvar, Madrid, 1 de febrero de 2008. 
MEI, II, Vol. 2, nº 2, pág. 110

RIKOWSKI, Ruth. “Globalización, bibliotecas, información y compromiso social”. En: De volcanes llena. Biblioteca y compromiso social. P. 159-212.

TORREMOCHA JIMÉNEZ, Alberto. "Biblioteca del Instituto Cervantes de Tetuán: de Menendez Pelayo a Vicente Aleixandre". Mi biblioteca: la revista del mundo bibliotecario. Año V, n. 16, invierno 2009, p. 88-95.

\section{Nota}

El artículo es una traducción y actualización de la ponencia presentada en la IFLA Satellite Meeting "Libraries and Society: an Pan-American Mediterranean Perspective", Palermo, 31st August-01st September, 2009.

http://www.iflamed.unipa.it/index.php/iflamed/2009/paper/viewFile/15/29 